\title{
Another gravitational solution found
}

New solutions of Einstein's equations are rarities, but the latest to appear has the particular interest of making the Big Bang less than a mathematical necessity.

EINSTEIN's theory of gravitation is generally (and correctly) regarded both as one of the great intellectual achievements of this century and as an awkward starting point for the solution of problems in the real world. The technical difficulty is that Einstein's theory is embodied in a set of non-linear differential equations (strictly, there are ten unknowns, all functions of space and time) which describe allowed patterns of the gravitational field. From any one may be inferred the corresponding distribution of mass or, more generally, energy to which that particular pattern corresponds.

Turning the problem around, so as to tell whether some particular distribution of mass is consistent with a solution of the equations, is difficult for at least two reasons. Because gravitational forces are long-range forces, even the most distant regions of the Universe contribute to local effects. (That is nothing but a reflection of the inverse-square law or of Mach's principle that the most distant galaxies contribute to the gravitational attraction between the Sun and the planets, for example.) Then, because the theory is a relativistic theory which mixes together space and time, not just a distribution of mass but its evolution in time must be specified before one can check whether it is a solution of the equations. One must conceive of a whole cosmology before one can check that it is plausible. It is a hit-ormiss business in which misses are more common than hits.

That is one reason why novel and useful solutions of Einstein's equations are celebrated as if they were new comets or supernovae. That, at least, is the mood engendered by nearly 75 years of frustration in the field.

It was not always thus. Right at the beginning, within a month of learning of Einstein's theory, in 1916, Swartzchild produced a solution of the equations that was not merely mathematically successful but which seemed to describe a reasonable and even realistic kind of Universe, one in which there is a spherically symmetrical distribution of mass. But, until the $1960 \mathrm{~s}$, there followed a long period in which more general solutions of Einstein's equations were as plentiful as water in a desert.

For the best part of this century, cosmologists have mostly had to be content with the family of solutions of Einstein's equations produced in 1925 by the Soviet mathematician Friedman, and which have become the foundation of FriedmanRobertson-Walker (called 'FRW') cosmologies. The underlying assumptions are simple enough. If the properties of space are the same in all directions (isotropic), Robertson and Walker first showed that the distribution of matter would also be isotropic; it fell to Friedman to show what this meant, mathematically, for the pattern of gravitational forces and thus for the distribution of matter and energy.

This is the mathematical basis of most cosmology. (The metric due to R. P. Kerr in 1963 is that on which speculations about isolated black holes are based, and is not strictly the basis for a cosmology.) FRW universes are not static, but are in general either expanding (which provides a natural interpretation of the observed red-shift of radiation from distant galaxies) or contracting. They are also isotropic, which is substantially borne out by observations of the real Universe - at least on sufficiently large scales, radio-galaxies, ordinary galaxies, the $\mathrm{X}$-ray background luminosity and the microwave background radiation appear to be isotropic within reasonable expectations of the measurement techniques. Strictly speaking, FRW universes need not be uniform in the sense that there is no systematic variation of the density of matter from one place to another, but it would then be necessary to suppose that the Earth, or at least the Galaxy, is uniquely placed, at odds with the spirit of the past 300 years.

But FRW universes have a number of special features, some of them mildly embarrassing. For one thing, with reasonable values of the mass density of the present Universe, they do now allow fluctuations of density in the early Universe to condense quickly enough into galaxies of the kind observed quickly enough to explain why the sky we see is filled with them. (That difficulty has been substantially turned by Guth's argument that there was a period of rapid expansion early in the history of the Universe.) A further difficulty is that all FRW universes have a singularity somewhere - if contracting, there comes a stage at which everything is concentrated at a point; if expanding, then they must have begun with something like the Big Bang which has become the contemporary creation legend.

It seems to be an open question whether other kinds of solutions of the equations might yield radically different but realistic universes. In particular, does the built-in assumption of isotropy force the conclusion that there is a singularity somewhere? A small army of people has by now done its best to explore small variations of FRW space-time, allowing for variations from strict isotropy, but, because these are necessarily approximations, the outcome is also necessarily muddy.

But is there not something to be said more generally? Whatever the difficulties of finding mathematical solutions for Einstein's equations, a feature of the theory of gravitation as distinctive as a Big Bang might be expected to be demonstrable directly, without the cumbersome intervention of algebra. Hawking and, independently, Penrose have argued that there must have been some singularity in the past history of the real Universe. But these arguments seem not to be safe against the discovery of a solution of the equations that lacks a singularity and which is at the same time realistic.

That is the promise of the latest development - the description of a new set of solutions of Einstein's field equations by José M. M. Senovilla from the University of Salamanca, who seems to have found a solution of Einstein's equations which describes a universe evolving from infinitely long ago to infinitely in the future without ever going through a condition recognizable as a Big Bang (Phys. Rev. Lett. 64, 2219; 1990).

Senovilla's universe, which has a kind of cylindrical symmetry, is a remarkably dull place. Time extends from infinity to infinity, with flat and empty space-time at each extreme. But, at times in the distant past, the density of matter and its pressure will increase steadily, until there comes a time when both quantities are a maximum, when the density will decrease again. If this corresponds to the Universe in which we live, the interesting time of maximum density already lies in the past. Whether the new solution is consistent with what is known of the Universe remains to be seen, while something unexpectedly odd may happen on the cylindrical axis of symmetry. But a demonstration that the Big Bang is not a mathematical necessity would be welcome in at least some quarters. So, too, should be Senovilla's proof that there is still a long way to go before the interest of solving Einstein's equations will be exhausted.

John Maddox 